\title{
Comportamiento del secado de tintas de plata para electrónica impresa
}

\section{Drying behavior of silver inks for printed electronics}

Presentación: 6-7/10/2020

\section{Doctorando:}

\section{Gabriel Maroli}

Laboratorio de Nanoelectrónica, Unidad de Investigación y Desarrollo de las Ingenierías, Facultad Regional Buenos Aires, Universidad Tecnológica Nacional - Argentina

Gabrielmaroli@gmail.com

\section{Director/a:}

\section{Felix Palumbo}

\section{Co-director/a:}

\section{María Alicia Volpe}

\section{Resumen}

La electrónica impresa propone un nuevo paradigma para el desarrollo de dispositivos flexibles. En particular, el método de impresión por chorro de tinta permite hacer prototipos de forma rápida y a muy bajo costo. Una de las claves de esta tecnología son las diferentes tintas con las que se puede trabajar, conductoras, dieléctricas y semiconductoras y sus procesos de sinterización. Para lograr las propiedades eléctricas deseadas, el sinterizado de las tintas es un paso fundamental. En este trabajo hemos desarrollado una tinta de plata, con la cual hemos conformado estructuras para su caracterización eléctrica, finalmente hemos propuesto un modelo de circuito del proceso de secado de tinta basado en datos experimentales y fisicoquímicos.

Palabras clave: Electrónica Impresa, Tintas Electrónicas, Microfabricación, Tinta de Plata

\begin{abstract}
Printed electronics proposes a new paradigm for the development of flexible devices. In particular, the inkjet printing method enables rapid prototyping at very low cost. One of the keys of this technology are the different inks that can be worked with, conductive, dielectric and semiconductor, and their sintering processes. To achieve the desired electrical properties, sintering is a critical step. In this work we have developed a silver ink, we have formed structures for their electrical characterization, finally we have proposed a circuit model of the ink drying process based on experimental and physicochemical data.
\end{abstract}

Keywords: Printed Electronic, Electronic inks, Microfabrication, Silver ink 


\section{Introducción}

La electrónica impresa (PE) es un término que define la impresión de circuitos en medios flexibles como papel, textiles, kapton, y una gran cantidad de medios potenciales(Kantola et al., 2009). PE fusiona la fabricación de dispositivos electrónicos y la impresión de texto/gráfica. Mediante esta combinación, se pueden fabricar productos electrónicos de alta calidad que son delgados, flexibles, portátiles, livianos, de diferentes tamaños, de bajo costo y amigables con el medio ambiente (Suganuma, 2014). Para la impresión por inyección de tinta es necesaria una serie de tintas de diferente naturaleza. La tinta conductora más utilizada se basa en plata (Venkata Krishna Rao et al., 2015). Esta tinta consiste en una suspensión coloidal de partículas de plata de tamaño nanométrico recubiertas con una fina capa de un polímero estabilizador. Debido a que las nanopartículas (NP) tienen una superficie específica muy alta, son muy propensas a sinterizarse a una temperatura relativamente baja, cerca del $10 \%$ al $20 \%$ de la temperatura de fusión del material a nivel macro) (Buffat \& Borel, 1976),(Gao \& Gu, 2015). Esta es la razón por la que las tintas metálicas se sinterizan a temperaturas tales como $100{ }^{\circ} \mathrm{C}$ o $150{ }^{\circ} \mathrm{C}$ (Moon et al., 2005), temperaturas mucho más bajas que el punto de fusión de la plata $\left(962{ }^{\circ} \mathrm{C}\right.$ ) (Somiya \& Moriyoshi, 1990). El curado térmico es un paso necesario para establecer el contacto eléctrico de las nanopartículas. Al elevar la temperatura por encima de la requerida para la evaporación del solvente, se inicia la eliminación del surfactante, logrando el contacto de las partículas metálicas(Greer \& Street, 2007). El proceso de sinterizado es crítico en el desempeño de las trazas conductora, no solo en lo que respecta a las propiedades eléctricas sino también a las mecánicas (Somiya et al., 1990). Algunos autores, como (Rodriguez, 2016), han desarrollado sus propias tintas; sin embargo, se trata de un proceso de prueba y error, en el que se pueden estimar algunos parámetros físicos de la tinta antes de su fabricación. El presente trabajo muestra el resultado del desarrollo de una tinta de plata ad hoc y su caracterización física y eléctrica. Además, este trabajo propone un modelo para predecir el comportamiento eléctrico de la tinta, curada por horno, antes de su fabricación. El modelo tiene la versatilidad de poder extrapolarlo a otros métodos de curado de tinta, como el curado óhmico o por infrarrojo. Esto es relevante para evaluar si la temperatura de sinterizado será compatible con el resto del proceso, especialmente con el sustrato.

\section{Desarrollo}

\section{A. Preparación de la tinta}

Para la tinta de plata, se utilizaron nanopartículas de plata (AgNP) de NanoTop, agua milli-Q, etanol y TritonMT X-100 de SigmaAldrich. Se vertieron agua y etanol en un vaso de precipitados con agitación constante y luego se agregaron lentamente los AgNP, asegurando una correcta homogeneización. Una vez que se ha añadido toda la masa de AgNP, se incorporó Triton MT X-100 y se dejó en agitación durante 2 horas. El producto final se agitó por ultrasonido utilizando un sonicador de punta durante 20 minutos (Rama et al., 2015). Se obtuvo una tinta de plata homogénea al $40 \% \mathrm{~m} / \mathrm{m}$.

\section{B. Conformación de la estructura TLM}

En primer lugar, se depositaron $400 \mu \mathrm{l}$ de tinta sobre un sustrato de vidrio de $25 \mathrm{~mm} \times 25 \mathrm{~mm}$ utilizando una micropipeta DragonLab de 100 a $1000 \mu$ l. Una vez depositada, la tinta se centrifugó a 1000 rpm durante 30 segundos con una aceleración de 250 $\mathrm{rpm} / \mathrm{s}$. Luego, la muestra se curó en un horno durante 1 hora a $150^{\circ} \mathrm{C}$. Posteriormente, se depositó una nueva capa de tinta de plata, repitiendo el proceso anterior. Este proceso se repitió 3 veces. Se llevó a cabo un recocido final a la misma temperatura durante $20 \mathrm{~h}$. A continuación, se conformó una estructura TLM (Transmission Line Measurement) sobre la superficie de plata mediante un proceso fotolitográfico. La técnica TLM se utiliza tradicionalmente para determinar la resistencia de contacto entre un metal y un semiconductor (Reeves \& Harrison, 1982), (Vinod, 2011). Sin embargo, en el contexto del presente trabajo se utilizó TLM para medir la resistencia de contacto entre la película de plata y la punta de la Probe Station. Para ello, se utilizó una fotorresina serie AZ 1500 de MicroChemical y una máscara impresa sobre filmina, con una resolución máxima de $100 \mu \mathrm{m}$. La figura 1 muestra la estructura de TLM de seis puntos obtenida, con un ancho de $1000 \mu \mathrm{m}$ y una separación entre los centros de los contactos de 850, 1000, 1250, 1500 y $1750 \mu \mathrm{m}$. 


\section{Imágenes TEM y SEM}

Se realizó el análisis de una gota de tinta utilizando un Microscopio Electrónico de Transmisión (TEM), Marca: JEOL, modelo 100 CX II, operado a un voltaje de aceleración de $100 \mathrm{kV}$ y con aumentos de $100000 \mathrm{x}$ y $270000 \mathrm{x}$ para determinar el tamaño de las nanopartículas(Fig.2). Las mediciones de tamaño se realizaron con el software Digital Micrograph. La información de tamaño de AgNPs es útil para estimar la capacitancia inicial de la tinta, que se analizará en una sección posterior. Adicionalmente, el proceso de sinterizado se observó mediante un Microscopio Electrónico de Barrido (SEM) durante el tiempo de secado (Fig. 3). La figura 2 muestra que la tinta tiene nanopartículas esféricas de tamaño medio de $100 \mathrm{~nm}$. Esta información es importante para comprender el comportamiento capacitivo de la tinta al comienzo del secado. El análisis SEM, que se muestra en la Fig. 3, podemos observar que durante el secado se forman caminos como raíces. Posteriormente, con el tiempo, la tinta se convierte en un film continuo.

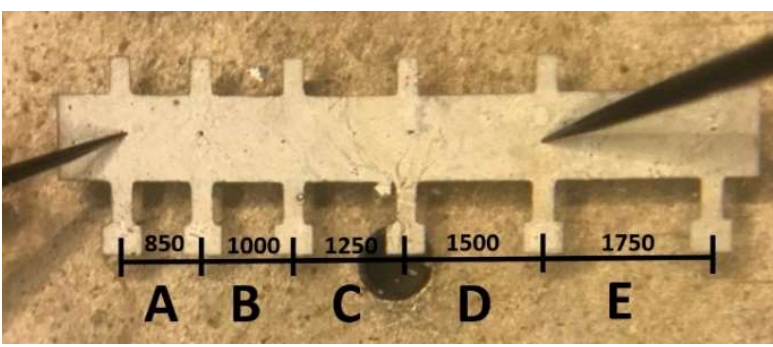

Fig 1. Estructura TLM realizada con tinta de plata sobre sustrato de vidrio, las distancias indicadas están en $\mu m$

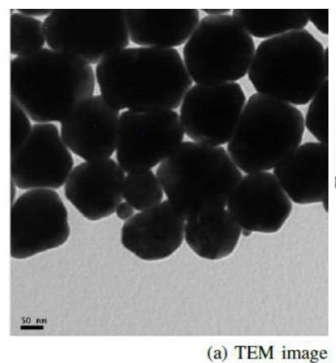

Fig 2. (a) Imagen TEM de nanopartículas de plata con amplificación de 100000x. (b) Distribución de tamaño de las nanopartículas.

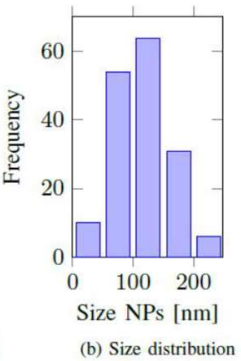

Fig 3. Imagen con escala de $10 \mu \mathrm{m}$ donde se puede observar la formación de los caminos conductores.

\section{Caracterización Eléctrica}

Se llevaron a cabo mediciones de corriente-voltaje (I-V) en los diferentes segmentos de la estructura TLM. El propósito de estas mediciones era determinar la resistencia de contacto y la resistividad de la tinta. Las tensiones aplicadas fueron de $-40 \mathrm{mV}$ a $100 \mathrm{mV}$ con la corriente limitada a $100 \mathrm{~mA}$. Las mediciones (I-V) se llevaron a cabo en oscuridad y en condiciones ambiente, usando una SMU Keithley 2636B. Para determinar el coeficiente térmico de la tinta, se repitieron estas mediciones para diferentes temperaturas: $100,125,150$ y $175^{\circ} \mathrm{C}$.

\section{Resultados}

La Fig. 4 muestra los resultados (I-V) de las mediciones realizadas a temperatura ambiente. Se puede observar una relación lineal para los diferentes segmentos: este comportamiento es el esperado para un material de naturaleza resistiva como lo es la tinta de plata una vez sinterizada. Cada segmento de la Fig. 4 es el promedio de 5 mediciones con una desviación estándar muy baja de alrededor del 0,55\%. Cada medición se caracterizó utilizando el valor de la pendiente, que representa la conductividad de la tinta. La Fig. 5 muestra la resistencia de cada segmento en función de la longitud y se construyó con la inversa de las líneas de la Fig. 4. La pendiente de la línea de tendencia en la Fig. 5 tiene unidades de $\Omega / \mathrm{mm}$ y corresponde a la resistencia lineal de la tinta. La ordenada al origen es 2 veces la resistencia de contacto entre la tinta y la punta de la Probe Station. La pendiente de la línea de tendencia es 1.27 $\times 10-2 \Omega /$ $\mathrm{mm}$ y la ordenada al origen es $1.05 \Omega$. Considerando los datos geométricos (Roberson et al., 2012) de nuestra estructura mencionados anteriormente, se puede concluir que la tinta tiene una resistividad de $19.03 \times 10-8 \Omega \times \mathrm{m}$, que es aproximadamente 11.9 veces mayor que la resistividad de la plata. Este valor es totalmente coherente con el informado por diferentes fabricantes para las tintas que se secan a igual temperatura (Sigma-Aldrich). En cuanto al comportamiento de la tinta en función de la temperatura, se puede ver en la Fig. 6 que también es lineal. Esto significa que la tinta obedece a la ecuación $\mathrm{R}=\mathrm{R} 0(1+\alpha \mathrm{T})$. En este caso, $\alpha=7.8 \times 10-3 \Omega /{ }^{\circ} \mathrm{C}$, que es aproximadamente dos veces mayor al de una barra de plata (Reuss, 2008).

El motivo de proponer un modelo es poder predecir el tiempo y la temperatura a la que debe secarse la tinta para alcanzar una determinada resistividad. Por otra parte, es igualmente importante poder estimar el tiempo necesario para que la tinta se 


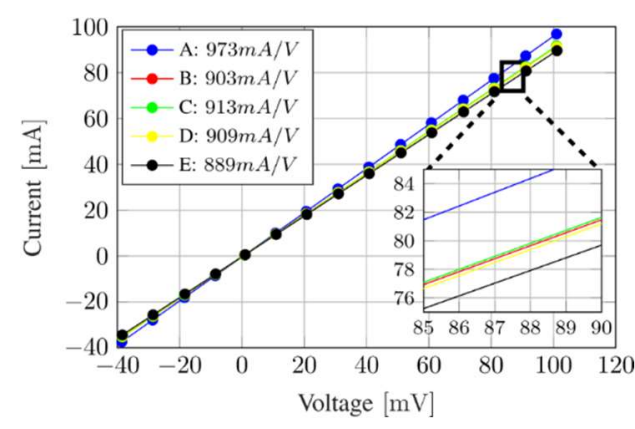

Fig 4. Curvas I-V para los diferentes segmentos de la estructura TLM

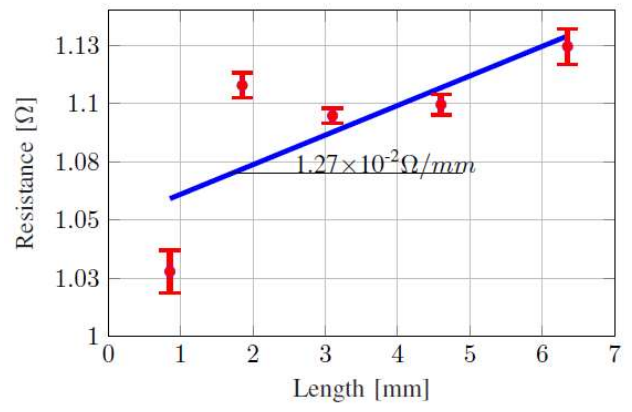

Fig 5. Relación entre la longitud del segmento y la resistencia medida. Las medidas mostraron dispersión, por eso se utilizó una línea de tendencia

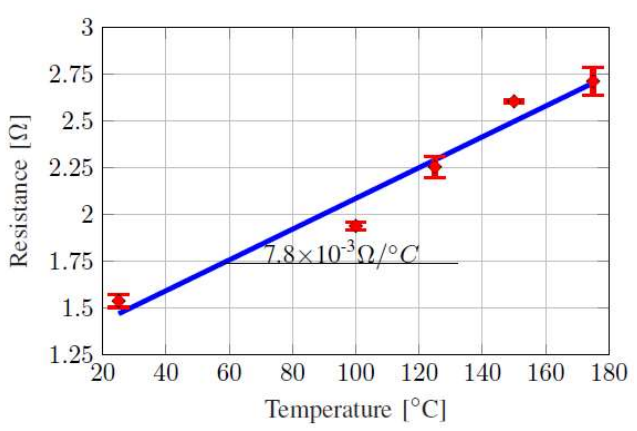

Fig 6. Relación lineal entre temperatura y resistencia medida

comporte de forma totalmente resistiva. Esta es una herramienta muy útil para predecir el comportamiento y la utilidad de una tinta casera. A partir de los datos obtenidos en el presente trabajo y la información recogida de los trabajos de Greer et al. (2007) y (Samano, 2017), se propone un modelo circuital para el secado de tintas en función del tiempo. La mayoría de los trabajos que abordan el secado de tintas, realizan mediciones en DC, obteniendo una resistividad que decrece exponencialmente.

Este fenómeno se puede explicar a partir del modelo difusivo propuesto por (Frenkel, 1945). En el trabajo de Samano (2017) se analiza la variación de la impedancia de la tinta, tanto en módulo como en fase, en función del tiempo para distintas frecuencias. A partir del comportamiento observado en Samano (2017), se propone un circuito simplificado RL serie en paralelo con un capacitor C.

Según este modelo,

$$
Z=\frac{j w L+R}{\left(1-w^{2} L C\right)+j w C R}
$$

La tinta fresca tiene una alta impedancia que varía desde unos pocos $\mathrm{G} \Omega$ hasta decenas de $M \Omega$, según la frecuencia. Además, se observa una fase capacitiva de $-80^{\circ}$ a altas frecuencias. De esta información se puede concluir que inicialmente la tinta es totalmente capacitiva, lo que concuerda con el hecho de que antes del tratamiento térmico, la tinta consta de pequeñas esferas muy próximas entre sí recubiertas por un dieléctrico. La capacitancia de cada esfera se puede estimar como $\mathrm{C}=\mathrm{r} / \mathrm{K}$, donde $\mathrm{r}$ es el radio de la esfera, estimado en $100 \mathrm{~nm}$ según la distribución de tamaño de la figura 2, y K es la constante de Coulomb $(8,987 \times 109 \mathrm{Nm} 2$ / C2 $)$. Cada AgNP aportará una capacitancia de 1,11 × 10-17 F. En la configuración propuesta, la superficie de contacto es de $2 \mathrm{~mm} \times 8 \mathrm{~mm}$ y la distancia entre contactos es de $50 \mathrm{~mm}$. Por lo tanto, se puede estimar una capacitancia inicial total considerando cada NP como un capacitor regular, colocado en paralelo en la placa de contacto y en serie a lo largo de la traza. La imagen TEM de la Fig. 2 muestra que las NPs tienden a agruparse, por lo tanto, para esta estimación pueden considerarse pegadas entre sí. Por tanto, la capacitancia inicial, $\mathrm{C} 0$, vendrá dada por:

$$
C_{0}=\frac{2 C_{0} 2 \times 10^{4} 8 \times 10^{4}}{5 \times 10^{5}}=7,12 \times 10^{-14} \mathrm{~F}
$$

Este valor capacitivo disminuye a medida que se evapora el dieléctrico que cubre las nanopartículas. Dado que el curado se realiza a una temperatura más baja que el punto de ebullición del dieléctrico, la tasa de evaporación es proporcional a la volatilidad de la sustancia, que a su vez es proporcional a la presión de vapor. La presión de vapor se puede calcular utilizando la ecuación de Antoine (Castellan, 1998); Las constantes de Antoine se obtuvieron mediante el simulador Hysys ${ }^{\circledR}$, generando un compuesto hipotético con la fórmula de Triton X-100. Finalmente, se obtuvo la siguiente expresión para la capacitancia:

$$
C(\mathrm{~T}, \mathrm{t})=\mathrm{C}_{0} \exp (-\beta(\mathrm{T}) \mathrm{t})
$$

donde $\beta\left(95^{\circ} \mathrm{C}\right)=0,39$. En a base a la fase, la impedancia y el valor de $\mathrm{C} 0$, se obtuvo el valor de $\mathrm{R} 0$.

$$
\theta=\tan ^{-1}\left(\frac{X_{c_{0}}}{R_{0}}\right)=-70^{\circ} \text { at } 10 \mathrm{KHz}
$$


Por lo tanto, $\mathrm{R} 0=80.6 \mathrm{M} \Omega$, un valor en línea con los reportados previamente (Rama et al., 2015). Basados en el modelo de Greer et al. (2007), sabemos que la resistividad tiene dos modelos de sinterizado, por lo que se obtuvo la siguiente expresión para la resistencia:

$$
\begin{aligned}
& R(T, t)=R_{0} \exp (-\gamma(T) t) \\
& R(T, t)=R_{1} \times \ln \left(\frac{a+a \times \sqrt{1-(5.77 t / \tau)^{(2 / 5)}}}{a-a \times \sqrt{1-(5.77 t / \tau)^{(2 / 5)}}}\right)
\end{aligned}
$$

donde $\tau$ es el tiempo característico de sinterizado por difusión de lattice (Coble, 1961), a $=100 \mathrm{~nm}$ es el tamaño de las nanopartículas y R1 $=\rho 0 \times$ Sl. La Eq. (5) se utiliza en los primeros 6 minutos y luego, cuando R5 $=\sim$ R6, se utiliza Eq. (6), de acuerdo con el modelo de Greer et al. (2007). La tinta fresca no tiene característica inductiva, por lo que $\mathrm{L}(0, \mathrm{~T})=0$. Asimismo, las pistas conductoras que dan lugar a la inductancia, se crean con la misma velocidad que desaparece la parte capacitiva. Por esa razón, tenemos que:

$$
L(T, t)=L_{0}[1-\exp (-\beta(T) t)]
$$

Luego, las trazas comienzan a unirse con la misma velocidad que disminuye la resistencia, dando como resultado una película continua. La expresión de la inductancia es:

$$
\mathrm{L}(\mathrm{T}, \mathrm{t})=\mathrm{L}_{0}[1-\exp (-\beta(\mathrm{T}) \mathrm{t})] \exp (-\gamma(\mathrm{T})(\mathrm{t}-\mathrm{td}))
$$

donde td es un retraso temporal y L0 $=5 \mathrm{mH}$ se determina a partir del pico de fase inductiva en el gráfico de Samano (2017). Después de reemplazar las Ecs. (3), (5) y (8) en la ecuación. (1), el modelo se simuló en Matlab® y se obtuvieron las Figs. 7 y 8 , mostrando módulo y fase. Después de 1200 minutos (20 horas), el modelo muestra una resistencia de aproximadamente $1.5 \Omega$ que es perfectamente compatible con nuestros datos experimentales. Además, podemos ver que tiene un comportamiento claramente resistivo, que cuadra con nuestros datos experimentales.

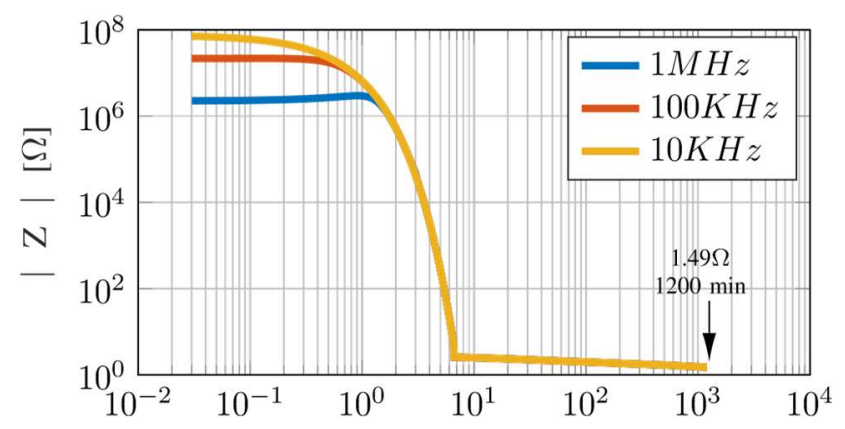

Fig 7. Simulación del Módulo de impedancia del modelo de secado en función del tiempo para 3 frecuencias diferentes

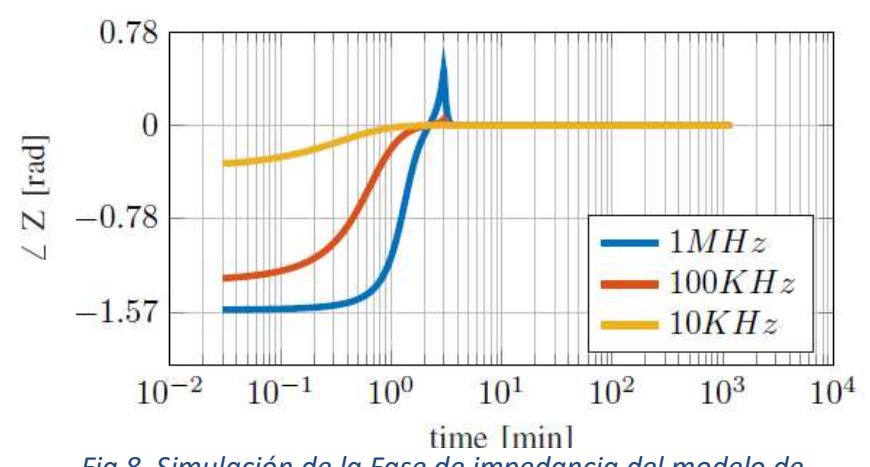

Fig 8. Simulación de la Fase de impedancia del modelo de secado en función del tiempo para 3 frecuencias diferentes

\section{Conclusiones}

En este artículo, hemos demostrado que es factible desarrollar una tinta ad hoc, partiendo de nanopartículas comerciales y generando la suspensión acuosa de las mismas. Como demuestra la caracterización física y eléctrica, nuestra tinta tiene propiedades comparables a las comerciales. Esto tiene un gran valor debido al bajo precio de las nanopartículas en relación con la tinta comercial. Hemos demostrado que un circuito simple puede modelar el secado de la tinta con gran fidelidad. En efecto, se pueden observar comportamientos capacitivos e inductivos a lo largo del proceso de secado, tanto en magnitud como en fase, de acuerdo con las mediciones reportadas en la literatura y con la física del secado. Es importante resaltar que conocer los parámetros físicos de una tinta, como el tamaño de las nanopartículas, su energía superficial, la difusividad, la naturaleza del dieléctrico que recubre las nanopartículas, su volatilidad y la volatilidad del solvente, nos permite simular el comportamiento de secado de la tinta a diferentes 
temperaturas. Otro punto para destacar es que este modelo no solo tiene relevancia teórica (nos permite correlacionar los cambios físicos que se producen en nuestra tinta durante el curado con el comportamiento eléctrico), sino que también tiene relevancia práctica, ya que también se puede utilizar para el curado de tintas por otros métodos. Para un curado óhmico o por infrarrojo, el modelo sería el mismo ya que el comportamiento físico no cambia, aunque los coeficientes Beta y Gamma deben recalcularse. El modelo también nos permitiría estimar cuándo la tinta tiene un comportamiento resistivo puro. Un modelo compacto para el proceso de sinterizado es útil para caracterizar la calidad de la tinta y optimizar el paso de sinterización. De esta forma, podemos determinar si nuestro sustrato y nuestro proceso son compatibles con estos parámetros. Eventualmente, la tinta se utilizará para conectar y producir dispositivos que formarán parte de un sistema más complejo. Si la caracterización de las trazas presenta un comportamiento reactivo, sabremos que la tinta no ha sido curada correctamente.

\section{Agradecimientos}

Este trabajo recibió financiamiento de las siguientes instituciones: UTN.BA bajo los proyectos CCUTIBA5219TC, CCUTIBA4764TC, CCUTNBA0005182, MATUNBA4936, CONICET bajo los proyectos PIP11220130100077CO y MINCyT bajo los proyectos PICT2016 / 0579 y PICT2017 / 2526. Agradecimiento especial a la Ing. Maria Julia Yánez del laboratorio de microscopía electrónica del Centro Científico Tecnologico - CONICET Bahía Blanca (CCTBB). Al Dr. Ing. Juan Jose Ortiz y Lic. Fabian De Vita de la Fundación Argentina de Nanotecnología. A PLAPIQUI-CONICET-UNS y al Departamento de Ingeniería Química de la UTN-FRBA que han proporcionado sus instalaciones para este desarrollo. Finalmente, a mis compañeros que componen el Laboratorio de Nanotecnología y que también han hecho su aporte, Ing. Fernando Aguirre, Lic. Santiago Boyeras, Ing. Sebastián Pazos y Dr. Ing. Hernan Giannetta.

\section{Referencias}

Buffat, P., \& Borel, J. P. (1976). Size effect on the melting temperature of gold particles. Physical Review A, 13(6), 2287-2298. https://doi.org/10.1103/PhysRevA.13.2287

Castellan, G. w. (1998). Physical Chemistry. Pearson.

Coble, R. L. (1961). Sintering crystalline solids. I. intermediate and final state diffusion models. Journal of Applied Physics, 32(5), 787-792. https://doi.org/10.1063/1.1736107

Frenkel, J. (1945). Viscous flow of crystalline bodies under the action of surface tension. Journal of Physics.

Gao, F., \& Gu, Z. (2015). Handbook of Nanoparticles. Handbook of Nanoparticles, iv, 1-25. https://doi.org/10.1007/978-3-319-13188-7

Greer, J. R., \& Street, R. A. (2007). Thermal cure effects on electrical performance of nanoparticle silver inks. Acta Materialia, 55(18), 6345-6349. https://doi.org/10.1016/j.actamat.2007.07.040

Kantola, V., Kulovesi, J., Lahti, L., Lin, R., Zavodchikova, M., \& Coatanéa, E. (2009). Printed Electronics, Now and Future. In Bit Bang - Rays to the Future.

Moon, K. S., Dong, H., Maric, R., Pothukuchi, S., Hunt, A., Li, Y. I., \& Wong, C. P. (2005). Thermal behavior of silver nanoparticles for lowtemperature interconnect applications. Journal of Electronic Materials, 34(2), 168-175. https://doi.org/10.1007/s11664-005-0229-8

Reeves, V., \& Harrison, H. B. (1982). Obtaining the Specific Contact Resistance from Transmission Line Model Measurements. IEE Electron Device Letters, 5(May), 111-113. https://doi.org/10.1109/EDL.1982.25502

Reuss, P. (2008). Neutron Physics. EDP Sciences.

Roberson, D. A., Wicker, R. B., \& MacDonald, E. (2012). Ohmic curing of printed silver conductive traces. Journal of Electronic Materials, 41(9), 2553-2566. https://doi.org/10.1007/s11664-012-2140-4

Rodriguez, M. B. (2016). Inkjet and screen printing for electronic applications. TDX (Tesis Doctorals En Xarxa), September, 1-225. www.tdx.cat Samano, A. (2017). Measurements of Conductive Film. Brunel University London.

Somiya, S., \& Moriyoshi, Y. (1990). Sintering Key Papers. In Sintering Key Papers. Elsevier Applied Science. https://doi.org/10.1007/978-94-0090741-6

Suganuma, K. (2014). Introduction to Printed Electronics. In Springer. https://doi.org/10.1007/978-1-4614-9625-0

Venkata Krishna Rao, R., Venkata Abhinav, K., Karthik, P. S., \& Singh, S. P. (2015). Conductive silver inks and their applications in printed and flexible electronics. RSC Advances, 5(95), 77760-77790. https://doi.org/10.1039/c5ra12013f

Vinod, P. N. (2011). Specific contact resistance measurements of the screen-printed Ag thick film contacts in the silicon solar cells by three-point probe methodology and TLM method. Journal of Materials Science: Materials in Electronics. https://doi.org/10.1007/s10854-011-0295-z 